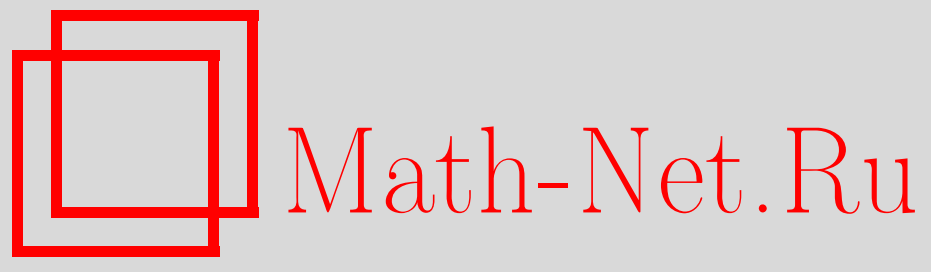

В. М. Бухштабер, Н. Рэй, Универсальный эквивариантный род и формула Кричевера, УМH, 2007, том 62, выпуск 1, 195-196

DOI: https://doi.org/10.4213/rm5695

Использование Общероссийского математического портала Math-Net.Ru подразумевает, что вы прочитали и согласны с пользовательским соглашением http://www.mathnet.ru/rus/agreement

Параметры загрузки:

IP : 3.82 .47 .9

26 апреля 2023 г., 17:24:22

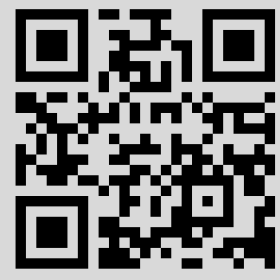




\section{Универсальный эквивариантный род и формула Кричевера}

\section{В. М. Бухштабер, Н. Рэй}

Мы будем рассматривать гладкие $2 n$-мерные многообразия $M$ с гладким действием $\theta$ окружности $S^{1}$ на них. Скажем, что $M$ обладает структурой нормалъно комплексного $S^{1}$-многообразия $\left(M, \theta, c_{\nu}\right)$, если задано эквивариантное вложение $i: M \rightarrow \mathbb{C}^{k}$ вместе с фиксированной эквивариантной комплексной структурой $c_{\nu}$ на нормальном расслоении $\nu(i)$. Используя стандартное действие $S^{1}$ на сфере $S^{2 m+1} \subset \mathbb{C}^{m+1}$, введем $2(m+n)$-мерное гладкое многообразие $W_{m}=S^{2 m+1} \times_{S^{1}} M$ и $k$-мерное комплексное векторное расслоение $q_{m}: E_{m} \rightarrow \mathbb{C} P^{m}$, где $E_{m}=S^{2 m+1} \times_{S^{1}} \mathbb{C}^{k}$. Тогда вложение $i$ продолжается до вложения $i^{\prime}: W_{m} \rightarrow E_{m}$, так что комплексная структура $c_{\nu}$ продолжается до комплексной структуры $c^{\prime}$ на нормальном расслоении $\nu\left(i^{\prime}\right)$.

Отображение $p_{m}: W_{m} \stackrel{i^{\prime}}{\longrightarrow} E_{m} \stackrel{q_{m}}{\longrightarrow} \mathbb{C} P^{m}$ является комплексно ориентированным (см. [1]) и задает класс комплексных кобордизмов $\Phi_{m}\left(M, \theta, c_{\nu}\right) \in U^{-2 n}\left(\mathbb{C} P^{m}\right)$. Ясно, что $\iota_{m}^{*} \Phi_{m+1}=\Phi_{m}$, где $\iota_{m}: \mathbb{C} P^{m} \rightarrow \mathbb{C} P^{m+1}$ - стандартное вложение. Обратная последовательность $\left(\Phi_{m}\left(M, \theta, c_{\nu}\right): m \geqslant 0\right)$ задает элемент $\Phi\left(M, \theta, c_{\nu}\right)$ из $\lim ^{-2 n}\left(\mathbb{C} P^{m}\right) \simeq$ $U^{-2 n}\left(\mathbb{C} P^{\infty}\right)$, который называется универсальным $S^{1}$-эквивариантным родом структуры $\left(M, \theta, c_{\nu}\right)$. Фундаментальные результаты о родах Хирцебруха многообразий с действием $S^{1}$ на его основе были получены в [2], [3]. Аналог рода $\Phi$ в случае компактной группы Ли $G$ изучается рядом авторов с 60-х годов (см. [4], [1]).

Имеет место изоморфизм $U^{*}\left(\mathbb{C} P^{\infty}\right) \cong \Omega^{*}[[u]]$, где $\Omega^{*}=U^{*}(p t)$ и $u$ - класс кобордизмов обратной последовательности $\left(\iota_{m}: m \geqslant 0\right)$. Задача - описать стабильно комплексные многообразия $G_{j}(M)$ размерности $2(n+j)$ такие, что $\Phi\left(M, \theta, c_{\nu}\right)=$ $\sum_{j=0}^{\infty}\left[G_{j}(M)\right] u^{j}$. Ясно, что $G_{0}$ есть $M$ со структурой $c_{\nu}$.

Рассмотрим $\left(S^{3}\right)^{j}=\left\{\left(y_{1}, z_{1} ; \ldots ; y_{j}, z_{j}\right) \in \mathbb{C}^{2 j}:\left|y_{i}\right|^{2}+\left|z_{i}\right|^{2}=1,1 \leqslant i \leqslant j\right\}$ с действием тора $T^{j}$ по формуле: $t \cdot\left(y_{1}, z_{1} ; \ldots ; y_{j}, z_{j}\right)=\left(t_{1} y_{1}, t_{1}^{-1} z_{1} ; \ldots ; t_{j} y_{j}, t_{j-1}^{-1} t_{j}^{-1} z_{j}\right)$, где $t=\left(t_{1}, \ldots, t_{j}\right)$. Фактормногообразие $B_{j}=\left(S^{3}\right)^{j} / T^{j}$ является $j$-й башней $S^{2}$ расслоений над $B_{0}=p t$, на которой имеются комплексные линейные расслоения $\eta_{1}, \ldots, \eta_{j}$ такие, что $E\left(\eta_{i}\right)=\left(S^{3}\right)^{j} \times_{T^{j}} \mathbb{C}$ относительно действия $t \cdot z=t_{i}^{-1} z$ для $z \in \mathbb{C}$. При $j>0$ изоморфизм $\tau\left(B_{j}\right) \oplus \mathbb{C}^{j} \cong\left(\bar{\eta}_{1} \oplus \eta_{1}\right) \oplus \cdots \oplus\left(\bar{\eta}_{j} \oplus \eta_{j-1} \eta_{j}\right)$ определяет стабильную комплексную структуру $c_{j}^{\partial}$ такую, что $B_{j}=\partial D_{j}$, где $D_{j}$ - пространство ассоциированного расслоения со слоем 3 -мерный диск, т.е. $\left[B_{j}\right]=0, j>0$.

Теорема 1. Фактормногообразие $\left(S^{3}\right)^{j} \times_{T^{j}} M$, где $T^{j}$ действует на $M$ по формуле $t \cdot x=\theta\left(t_{j}\right) \cdot x$, задает многообразие $G_{j}(M)$ для всех $j \geqslant 0$. Стабильно комплексная структура $c(j)$ на $G_{j}(M)$ индуцирована расслоением $M \rightarrow G_{j}(M) \rightarrow B_{j}$.

Доказательство использует $[1 ; 1.7]$ и тот факт, что классифицирующие отображения векторных расслоений $\eta_{j} \rightarrow B_{j}$ дают базис в $U_{*}\left(\mathbb{C} P^{\infty}\right)$, двойственный базису $u^{k}$, $k=0,1,2, \ldots$, в $U^{*}\left(\mathbb{C} P^{\infty}\right)[5 ;$ утверждение 2.2$]$.

ЗАмечание 2. Очевидно, что наши $G_{j}\left(M^{2 n}\right)$ комплексно бордантны несвязным многообразиям $\gamma^{j}\left(M^{2 n}\right)$ из [6; теорема 6.3]. Таким образом, мы показали, что на геометрических классах гомоморфизм пополнения кольца гомотопических бордизмов $M U_{*}^{S_{1}}$ по идеалу аугментации (см. [7; гл. 27]) совпадает с $\Phi$. Более того, $G_{j}\left(M^{2 n}\right)$ диффеоморфны многообразиям $M^{2(n+j)}$ из [8] и тем самым индуцируют на них стабильно комплексную структуру.

Скажем, что $M$ обладает структурой тангенииалъно стабильно комплексного $S^{1}$-многообразия $\left(M, \theta, c_{\tau}\right)$, если заданы некоторое комплексное расслоение $\xi \rightarrow M$ и изоморфизм

$$
c_{\tau}: \tau(M) \oplus \mathbb{C}^{l-n} \rightarrow \xi
$$

где $\tau(M)$ - касательное расслоение, такие, что композиция

$$
\rho(t): \xi \stackrel{c_{\tau}^{-1}}{\longrightarrow} \tau(M) \oplus \mathbb{C}^{l-n} \stackrel{d \theta(t) \oplus I}{\longrightarrow} \tau(M) \oplus \mathbb{C}^{l-n} \stackrel{c_{\tau}}{\longrightarrow} \xi, \quad t \in S^{1},
$$


является комплексным преобразованием для каждого $t \in S^{1}$, где $d \theta(t)$ - дифференциал преобразования $\theta(t)$. Таким образом, (2) задает представление $\rho: S^{1} \rightarrow$ $\operatorname{Hom}_{\mathbb{C}}(\xi, \xi)$. С точностью до естественной эквивалентности, структура $\left(M, \theta, c_{\tau}\right)$ onpeделяет структуру $\left(M, \theta, c_{\nu}\right)$, и поэтому можно ввести $\Phi\left(M, \theta, c_{\tau}\right)$ как $\Phi\left(M, \theta, c_{\nu}\right)$ для соответствующей структуры $c_{\nu}$. С другой стороны, $c_{\nu}$ не всегда определяет эквивариантную $c_{\tau}[7 ;$ гл. 28].

Пусть $x \in M$ - изолированная неподвижная точка действия $\theta$. Тогда ассоциированное представление $\rho_{x}: S^{1} \rightarrow G L(l, \mathbb{C})$ разлагает слой $\xi_{x} \cong \mathbb{C}^{l}$ в сумму $\mathbb{C}^{n} \oplus \mathbb{C}^{l-n}$, где $\rho_{x}$ действует (без неподвижных точек) как $\rho_{x, 1} \oplus \cdots \oplus \rho_{x, n}$ на $\mathbb{C}^{n}$ и тривиально на $\mathbb{C}^{l-n}$. Здесь $\rho_{x, j}$ - одномерное представление веса $\omega_{x, j}$, т.е. $\rho_{x, j}(t) v=t^{\omega_{x, j}} v$. Таким образом, каждой изолированной неподвижной точке $x$ сопоставляется набор весов $\omega_{x}=\left(\omega_{x, 1}, \ldots, \omega_{x, n}\right)$. Более того, изоморфизм $c_{x, \tau}$ (см. (1)) индуцирует ориентацию в касательном пространстве $\tau_{x}$, используя которую мы вводим $\sigma(x)$ как знак детерминанта линейного отображения

$$
\tau_{x}(M) \stackrel{I \oplus 0}{\longrightarrow} \tau_{x}(M) \oplus \mathbb{C}^{l-n} \stackrel{c_{\tau, x}}{\longrightarrow} \xi_{x} \cong \mathbb{C}^{n} \oplus \mathbb{C}^{l-n} \stackrel{\pi}{\longrightarrow} \mathbb{C}^{n},
$$

где $\pi$ - проекция на первое слагаемое. Знак важен в торической топологии [9].

Пример 3. $S^{1}$-многообразие $\left(B_{1}=S^{2}, \theta, c_{1}^{\partial}\right)$, где $\theta(t)\left[z_{1}: z_{2}\right]=\left[t z_{1}: z_{2}\right]$ имеет неподвижные точки $x_{1}=[1,0]$ и $x_{2}=[0,1]$ с весами $\omega_{x_{1}, 1}=\omega_{x_{2}, 1}=1$ и знаками $\sigma\left(x_{1}\right)=-\sigma\left(x_{2}\right)=1$.

Пусть $\left\{[q](u) \in \Omega^{*}[[u]]: q \in \mathbb{Z}\right\}$ - степенная система формальной группы $f(u, v)$ в комплексных кобордизмах (см. [10]). Ряд $[q](u)=q u \bmod \left(u^{2}\right)$ однозначно задается соотношениями $[0](u)=0$ и $[q](u)=f(u,[q-1](u))$ для всех $q \in \mathbb{Z}$.

Теорема 4. Если множество $X$ неподвижных точек действия $\theta$ конечно, то в $U^{-2 n}\left(\mathbb{C} P^{\infty}\right)$ имеет место формула

$$
\Phi\left(M, \theta, c_{\tau}\right)=\sum_{x \in X} \sigma(x) \prod_{i=1}^{n} \frac{1}{\left[\omega_{x, i}\right](u)} .
$$

Доказательство использует утверждение 3.8 из [1] и построение $\sigma(x)$, см. выше.

ЗАмечание 5. Для почти комплексного $S^{1}$-многообразия $M$ мы можем положить $l=n$ в (1). Тогда $\sigma(x)=1$ для всех $x \in X$ и формула (3) переходит в формулу Кричевера, [2; (2.7)].

Теоремы 1 и 4 очевидным образом переформулируются для случая эффективного действия тора $T^{k}$ на $2 n$-мерном многообразии, $k \leqslant n$.

\section{Список литературы}

[1] D. Quillen, Adv. Math., 7:1 (1971), 29-56. [2] И. М. Кричевер, Изв. АН СССР. Сер. матем., 38:6 (1974), 1289-1304. [3] И. М. Кричевер, Матем. заметки, 47:2 (1990), 34-45, 158. [4] T. tom Dieck, Topology, 9:4 (1970), 345-358. [5] N. Ray, Proc. Edinb. Math. Soc. (2), 29:3 (1986), 413-422. [6] D. P. Sinha, Amer. J. Math., 123:4 (2001), 577-605. [7] J. P. May (ed.), Equivariant homotopy and cohomology theory, CBMS Reg. Conf. Ser. Math., 91, Amer. Math. Soc., Providence, RI, 1996. [8] П. Коннер, Э. Флойд, Гладкие периодические отображения, Мир, М., 1969. [9] V. M. Buchstaber, T. E. Panov, N. Ray, Mosc. Math. J., 7:2 (2007). [10] С. П. Новиков, Изв. АН СССР. Сер. матем., 31:4 (1967), 855-951.

\section{B. M. Бухштабер (V. M. Buchstaber)}

Математический институт им. В. А. Стеклова РАН

E-mail: buchstab@mi.ras.ru

H. Рэй (N. Ray)

School of Mathematics, University of Manchester, England

E-mail: nige@ma.man.ac.uk
Представлено С. П. Новиковым Принято редколлегией 07.12 .2006 